\title{
Validation of the pepsinogen test method for gastric cancer screening using a follow-up study
}

\author{
Shigeto Mizuno ${ }^{1}$, Masao Kobayashi ${ }^{2}$, Shohken Tomita ${ }^{3}$, Ikuya Miki ${ }^{1}$, Atsuhiro Masuda ${ }^{1}$, Mitsuko Onoyama ${ }^{1}$, \\ Yasuki Habu ${ }^{4}$, Hideto Inokuchi ${ }^{5}$, and Yoshiyuki Watanabe ${ }^{6}$ \\ ${ }^{1}$ Department of Medical Pharmaceutics, Kobe Pharmaceutical University, Kobe, Japan \\ ${ }^{2}$ Department of Health Care, Kyoto Second Red Cross Hospital, Kyoto, Japan \\ ${ }^{3}$ Kansai Occupational Health Association, Osaka, Japan \\ ${ }^{4}$ Department of Gastroenterology, Saiseikai Noe Hospital, Osaka, Japan \\ ${ }^{5}$ Department of Gastroenterology, Hyogo Cancer Center, Akashi, Japan \\ ${ }^{6}$ Department of Epidemiology for Community Health and Medicine, Kyoto Prefectural University of Medicine Graduate School of Medical \\ Science, 465 Kajii-cho, Kawaramachi-Hirokoji, Kamigyo-ku, Kyoto 602-8566, Japan
}

\begin{abstract}
Background. Serum pepsinogen (PG) measurement has been used for gastric cancer screening since the 1990s. However, there are no reports comparing the screening validity of the PG test method with that of conventional X-ray examination directly in the same population, using a follow-up study.

Methods. From April 2000 to March 2001, 12120 residents of Osaka Prefecture, who underwent opportunistic screening at a medical checkup organization in Osaka city (hereafter, "the organization"), were enrolled. They received both a barium meal examination and PG test simultaneously. All the participants were followed up for a 1-year period after the screening. For the participants advised to undergo endoscopic examination, the results of those who were examined at the organization were tallied. The other participants were checked using the Osaka Cancer Registry (hereafter, "the registry").

Results. Of the 12120 participants, $493(4.1 \%)$ were positive with the PG method and $728(6.0 \%)$ were positive with the X-ray method. Fifty-four $(0.4 \%)$ were positive for both methods. Thirteen gastric cancer cases were diagnosed by successive esophagogastroduodenoscopies at the organization. Six additional gastric cancer cases were identified by record linkage with the registry. The sensitivity, specificity, and positive predictive values of the PG method with a PGI cutoff level of $\leq \mathbf{3 0} \mathrm{ng} / \mathrm{ml}$ and PGI/ PGII ratio of $\leq \mathbf{2 . 0}$ were $36.8 \%, 96.0 \%$, and $1.4 \%$, respectively. These values for the direct $X$-ray examination were $68.4 \%, 94.1 \%$, and $1.8 \%$, respectively.

Conclusion. The PG test method alone with a PGI cutoff level of $\leq 30 \mathrm{ng} / \mathrm{ml}$ and PGI/PGII $\leq 2.0$ is not appropriate for gastric cancer screening.
\end{abstract}

Key words Pepsinogen - Gastric cancer - Screening · Followup study

\section{Introduction}

The mortality rate from gastric cancer has continued to decrease since the 1960s; however, it still remains the second major cause of cancer death in Japan. Thus, continuous social measures are still needed for the early detection of gastric cancer [1].

Screening for gastric cancer with photofluorography, which was introduced in the 1960s, is an epidemiologically effective method of reducing the mortality rate of gastric cancer [2,3]. Recently, however, some problems have been pointed out; there have been decreases in efficiency, decreased coverage rates, and decreased new participants in gastric cancer screening.

On the other hand, the measurement of serum pepsinogen (PG) I and PGII levels, which was developed as a serological marker for the diagnosis of chronic atrophic gastritis, an accepted background factor of gastric cancer, by Samloff et al. [4] and Miki et al. in the 1980s [5], was introduced as a screening method for gastric cancer in the 1990s [6-9].

Although there have been many cross-sectional reports of the validity of the PG test method, there are no reports comparing the screening validity of the PG test method with that of conventional X-ray examination (a standard screening method for stomach cancer in Japan), directly in the same population, using a follow-up study. Therefore, we conducted a follow-up study to reveal the validity of both the PG test method and X-ray examination in the same population in a health checkup setting in Japan. 


\section{Subjects and methods}

\section{Subjects}

From April 2000 to March 2001, 12120 residents (7590 male, 4530 female) of Osaka Prefecture, who underwent opportunistic screening at a medical checkup organization (hereafter, "the organization") in Osaka city, were enrolled in this study. They received both a barium meal examination and a serum PG test simultaneously as screening for gastric cancer. Subjects who had renal dysfunction or a history of gastrectomy were excluded from the study.

\section{Methods}

In the organization, individuals with a PGI level of $\leq 30 \mathrm{ng} / \mathrm{ml}$ and a PGI/PGII ratio of $\leq 2.0$ were advised to undergo esophagogastroduodenoscopy (EGD). Those with abnormal X-ray findings, such as elevated or depressed lesions of the stomach, were also advised to undergo EGD. Serum PG levels were measured using the ARCHITECT PGI \& PGII assay, a chemiluminescent microparticle immunoassay (Abbott Laboratories, Chicago, IL, USA). The PG test for gastric cancer screening was first introduced in 2000 at the organization. X-ray examination with direct radiography was performed using the double-contrast barium meal method adopted at most Japanese medical institutions that conduct arbitrary checkups.

All participants were followed up for a 1-year period after screening. Of the participants advised to undergo endoscopic examination, the results of those who were examined at the same organization were tallied. The other participants were checked with the Osaka Cancer Registry in January 2006, when the registration was fixed up to March 2002, in order to identify the incidence of stomach cancer. As a general rule, all patients diagnosed with gastric cancer, including early cancer, at any hospital in Osaka prefecture are registered with the registry. The sensitivity, specificity, and positive predictive value (PPV) with 95\% confidence intervals (CIs) for the PG test method, with a PGI cutoff level of $\leq 30 \mathrm{ng} / \mathrm{ml}$ and PGI/PGII ratio of $\leq 2.0$, and X-ray examination to diagnose stomach cancer were calculated. The protocol of this study was approved by the ethics board of Kyoto Prefectural University of Medicine.

\section{Results}

Two-thirds of the subjects were male. The age of the 12120 subjects ranged from 15 to 84 years (median, 50 years) in the male group and from 22 to 84 years
Table 1. Study subjects

\begin{tabular}{lrrr}
\hline Age (years) & Male & Female & Total \\
\hline$\leq 29$ & 40 & 38 & 78 \\
$30-39$ & 1449 & 733 & 2182 \\
$40-49$ & 2510 & 1490 & 4000 \\
$50-59$ & 2425 & 1423 & 3848 \\
$60-69$ & 966 & 709 & 1675 \\
$70-79$ & 187 & 130 & 317 \\
$80-85$ & 13 & 7 & 20 \\
Total & 7590 & 4530 & 12120 \\
Median age (years) & 50 & 49 & \\
\hline
\end{tabular}

(median, 49 years) in the female group. Subjects (both male and female) in their forties made up the highest number among age brackets. There were 9860 subjects $(81.4 \%)$ older than 40 years (Table 1). Of the 12120 subjects, 3045 (25.1\%) had undergone a medical checkup for the first time, 1579 persons $(13.0 \%)$ for the second time, and 7496 persons $(61.9 \%)$ for the third time or more.

Of the 12120 subjects who participated in this study, $493(4.1 \%)$ met the criterion of the organization for a positive PG test and 728 participants $(6.0 \%)$ were found to have abnormal findings on the direct X-ray examination of the stomach. Fifty-four subjects $(0.4 \%)$ were positive for both methods.

All gastric cancer cases included in the results of the two screening tests, and the clinical features of gastric cancer, are shown in Table 2. Thirteen gastric cancer cases ( 2 advanced type and 11 early type) were diagnosed by successive EGDs at the same organization. An additional 6 gastric cancer cases (1 advanced type, 4 early type, and 1 undetermined type with neighboring lymph node metastasis) were identified by the record linkage with the Osaka Cancer Registry. These 6 cases were registered by hospitals in Osaka and did not include cases with death certificate only (DCO), that is, cases identified solely from death certificates.

The sensitivity, specificity, and PPV of the PG test method with a PGI cutoff level of $\leq 30 \mathrm{ng} / \mathrm{ml}$ and PGI/ PGII $\leq 2.0$, the criteria for recommendation of a further examination, for all participants were $36.8 \%$ (95\% CI, $15.2 \%-58.5 \%$ ), $96.0 \%$ (95\% CI, 95.6\%-96.3\%), and $1.4 \%$ (95\% CI, $0.38 \%-2.46 \%)$, respectively. These values for the direct X-ray examination were $68.4 \%$ (95\% CI, 47.5\%-89.3\%), 94.1\% (95\% CI, 93.7\%$94.5 \%$ ), and $1.8 \%$ (95\% CI, $0.82 \%-2.75 \%$ ), respectively (Table 3). For participants who had received a checkup for the first time, the sensitivity, specificity, and PPV of the PG test method with a PGI cutoff level of $\leq 30 \mathrm{ng} / \mathrm{ml}$ and PGI/PGII $\leq 2.0$ were $33.3 \%, 97.3 \%$, and $1.2 \%$, respectively. These values for the direct X-ray examination were $33.3 \%, 92.2 \%$, and $0.4 \%$, respectively. 
Table 2. Characterization of gastric cancer cases

\begin{tabular}{|c|c|c|c|c|c|c|c|c|c|c|}
\hline $\begin{array}{l}\text { Case } \\
\text { no. }\end{array}$ & $\begin{array}{c}\text { Age } \\
\text { (years) }\end{array}$ & Sex & X-ray & $\begin{array}{c}\text { PGI } \\
(\mathrm{ng} / \mathrm{ml})\end{array}$ & $\begin{array}{c}\text { PGII } \\
(\mathrm{ng} / \mathrm{ml})\end{array}$ & PGI/II & PG & Type & Histology & Locus \\
\hline 1 & 43 & M & + & 52.1 & 11.8 & 4.4 & - & Early & Diffuse & Body \\
\hline 2 & 49 & M & + & 78.1 & 13.4 & 5.8 & - & Early & Intestinal & Antrum \\
\hline 3 & 57 & M & + & 48.2 & 4.2 & 11.5 & - & Early & Intestinal & Cardia \\
\hline 4 & 47 & M & + & 53.7 & 11.6 & 4.6 & - & Early & Diffuse & Antrum \\
\hline 5 & 66 & $\mathrm{~F}$ & - & 5.8 & 7.8 & 0.7 & $2+$ & Early & Intestinal & Body \\
\hline 6 & 69 & $\mathrm{~F}$ & - & 16.8 & 10.9 & 1.5 & $2+$ & Early & Intestinal & Body \\
\hline 7 & 57 & M & + & 17.0 & 9.1 & 1.9 & $2+$ & Early & Intestinal & Body \\
\hline 8 & 72 & $\mathrm{M}$ & + & 3.5 & 3.6 & 1.0 & $2+$ & Early & Intestinal & Antrum \\
\hline 9 & 64 & $\mathrm{~F}$ & + & 68.3 & 24.6 & 2.8 & + & Early & Diffuse & Body \\
\hline 10 & 48 & M & + & 13.4 & 20.9 & 0.6 & $2+$ & Early & Intestinal & Body \\
\hline 11 & 57 & M & + & 33.3 & 18.3 & 1.8 & + & Advanced & Diffuse & Body \\
\hline 12 & 54 & M & + & 12.4 & 13.9 & 0.9 & $2+$ & Advanced & Diffuse & Antrum \\
\hline 13 & 54 & M & + & 31.2 & 18.0 & 1.7 & + & Early & Intestinal & Body \\
\hline $14^{\mathrm{a}}$ & 76 & $\mathrm{M}$ & - & 76.0 & 5.2 & 14.6 & - & Early & NA & Antrum \\
\hline $15^{\mathrm{a}}$ & 58 & M & - & 27.9 & 3.6 & 7.8 & - & Early & NA & NA \\
\hline $16^{\mathrm{a}}$ & 64 & M & - & 33.6 & 19.1 & 1.8 & + & Early & NA & Body \\
\hline $17^{\mathrm{a}}$ & 66 & M & - & 16.2 & 7.9 & 2.1 & + & Early & NA & NA \\
\hline $18^{\mathrm{a}}$ & 40 & M & + & 30.0 & 11.7 & 2.6 & + & NA & NA & NA \\
\hline $19^{\mathrm{a}}$ & 53 & $\mathrm{M}$ & + & 10.5 & 9.1 & 1.2 & $2+$ & Advanced & NA & Body \\
\hline
\end{tabular}

X-ray -, normal X-ray finding; X-ray +, abnormal X-ray finding; PG, (pepsinogen) -, PGI > $70 \mathrm{ng} / \mathrm{ml}$ or PGI/PGII $>3.0$; PG +, PGI $\leq 70 \mathrm{ng} / \mathrm{ml}$ and PGI/PGII $\leq 3.0$, excluding PG 2+; PG 2+, PGI $\leq 30 \mathrm{ng} / \mathrm{ml}$ and PGI/PGII $\leq 2.0$ (positive criterion of the organization); NA, not available

${ }^{a}$ Identified by the Osaka Cancer Registry

Table 3. Results of gastric cancer screening and follow-up study

\begin{tabular}{|c|c|c|c|}
\hline & \multicolumn{2}{|c|}{ Pepsinogen method } & \multirow[b]{2}{*}{ Total } \\
\hline & $2+$ &,+- & \\
\hline \multicolumn{4}{|c|}{ Gastric cancer } \\
\hline Positive & 7 & 12 & 19 \\
\hline Negative & 486 & 11615 & 12101 \\
\hline Total & 493 & 11627 & 12120 \\
\hline \multicolumn{4}{|c|}{$\begin{array}{l}-, \text { PGI }>70 \mathrm{ng} / \mathrm{ml} \text { or PGI/PGII }>3.0 ;+, \mathrm{PGI} \leq 70 \mathrm{ng} / \mathrm{ml} \text { and } \mathrm{PGI} / \mathrm{PGII} \\
\leq 3.0 \text {, excluding } 2+; 2+, \mathrm{PGI} \leq 30 \mathrm{ng} / \mathrm{ml} \text { and } \mathrm{PGI} / \mathrm{PGII} \leq 2.0 \text { (positive } \\
\text { criterion of the organization) }\end{array}$} \\
\hline & \multicolumn{2}{|c|}{ X-ray method } & \\
\hline & Positive & Negative & Total \\
\hline \multicolumn{4}{|c|}{ Gastric cancer } \\
\hline Positive & 13 & 6 & 19 \\
\hline Negative & 715 & 11386 & 12101 \\
\hline Total & 728 & 11392 & 12120 \\
\hline
\end{tabular}

\section{Discussion}

In the present study, the PG test method with a PGI cutoff level of $\leq 30 \mathrm{ng} / \mathrm{ml}$ and PGI/PGII ratio of $\leq 2.0$, the criterion adopted for gastric cancer screening by the organization, showed a relatively lower level of sensitivity, a similar level of specificity, and a similar PPV compared with the direct X-ray method.
A screening program with an upper gastrointestinal series has been confirmed as an effective method in reducing the mortality from gastric cancer in Japan [10]. Since the X-ray method with photofluorography was first introduced for stomach cancer screening in the 1960s, it has played a key role in gastric cancer screening in both the settings of population-based screening as public policy and opportunistic screening [11]. About 140000 inhabitants participated in population-based gastric cancer screening by the X-ray method in 1982 . The participants increased year by year in the 1980s and have numbered about 4 million since the 1990s [12]. The approximate sensitivity, specificity, and PPV of the Xray method were reported to be $70 \%-80 \%, 90 \%$, and $0.7 \%-2.0 \%$, respectively [13-18].

However, a decrease in the efficiency of the conventional gastric cancer screening has recently been pointed out. Because people eschew such uncomfortable sideeffects of the X-ray method as constipation and ileus due to the barium meal, the number of participants in the program has hovered at around $13 \%$ of the target population. Furthermore, it is necessary to pay attention to the fact that the infection rate of Helicobacter pylori, one definitive cause of gastric cancer, is known to have fallen recently, particularly in the younger generation. Effective gastric cancer screening cannot be achieved by the conventional method that advises all inhabitants who have reached the appropriate age to undergo X-ray examination. To continue gastric cancer screening in the future, it is important to focus on the 
groups at high risk of gastric cancer and to centralize the examination system, one of the social resources, accordingly.

Chronic atrophic gastritis caused by Helicobacter pylori infection is thought to be the predominant risk factor for gastric carcinogenesis [19-21]. Especially, intestinal-type gastric cancer located in the gastric antrum has a stepwise carcinogenic process; chronic atrophic gastritis-intestinal metaplasia-dysplasiagastric cancer. Endoscopic findings are useful for making the diagnosis of chronic atrophic gastritis. Kimura [22] has reported that gastric mucosal atrophy progresses from the pyloric side to the oral side with age.

In the 1980s, Samloff et al. [4] and Miki et al. [5] reported that an estimate of the extent of gastric mucosal atrophy was possible by measuring serum PGI and PGII levels. Although PGI is secreted from the fundic gland region of the stomach, PGII is secreted from the fundic and pyloric gland regions of the stomach and also from the duodenum $[23,24]$. When mucosal atrophy advances from the pyloric gland region towards the oral side, as in the process of chronic atrophic gastritis, the PGI level and PGI/PGII ratio decrease progressively. A trial to use the serum PG level for gastric cancer screening was started in the 1990s, based on this principle. Previous studies have reported that the sensitivity and specificity of the PG method were $56.8 \%-88.5 \%$ and $81.3 \%-$ $92.0 \%$, respectively [25-29].

Currently, either of the two methods, or both, have been used as population-based and opportunistic screening examinations for gastric cancer. However, although there have been many cross-sectional reports of the validity of the PG test, there have been no reports comparing the screening validity of the PG test method with that of the conventional X-ray examination directly in the same population, using a follow-up study.

Therefore, we adopted a follow-up method in the present study. The reliability of validation may be decreased because of the occurrence of false-negatives when the validity of screening methods is tested only for subjects with positive results and for those who undergo thorough examinations sequentially. We were able to assess the efficacy of two different screening methods precisely, because we verified the incidence of gastric cancer using follow-up data on the 12120 participants who were tested by both the PG method and the X-ray method simultaneously. Specifically, we cross-checked the participants in opportunistic screening at a medical checkup organization in Osaka city to assess whether stomach cancer occurred for 1 year after examination, by means of a record linkage to the Osaka Cancer Registry. From verification in the registry, we were able to identify 6 gastric cancer cases in addition to the 13 cases diagnosed by successive EGD at the same organization.
Until now, the three main positive criteria for the PG test method used for gastric cancer screening in Japan have been: $\mathrm{PGI} \leq 70 \mathrm{ng} / \mathrm{ml}$ and $\mathrm{PGI} / \mathrm{PGII} \leq 3.0 ; \mathrm{PGI} \leq$ $50 \mathrm{ng} / \mathrm{ml}$ and PGI/PGII $\leq 3.0$; and $\mathrm{PGI} \leq 30 \mathrm{ng} / \mathrm{ml}$ and $\mathrm{PGI} / \mathrm{PGII} \leq 2.0$. The last criterion reflects the most severe gastric mucosal atrophy, and the highest risk for developing gastric cancer. As sensitivity deteriorates and specificity rises in the order of the positive criteria mentioned before, Miki et al. [29] have recommended the criteria of PGI $\leq 70 \mathrm{ng} / \mathrm{ml}$ and $\mathrm{PGI} / \mathrm{PGII} \leq 3.0 \mathrm{in}$ screening for gastric cancer. The organization adopted the strictest criterion partly because it was difficult to perform EGD in a larger number of subjects, considering cost-effectiveness and the limited capacity of the organization, and also because the PG test was added to the existing X-ray screening. In fact, two gastric cancer cases were detected additionally with the PG method. However, the sensitivity of 36.8\% (95\% CI, $15.2 \%-58.5 \%$ ) with a PGI cutoff level of $\leq 30 \mathrm{ng} / \mathrm{ml}$ and $\mathrm{PGI} / \mathrm{PGII} \leq 2.0$ is not enough for a single screening test.

In comparison, when we calculated the same values with a PGI cutoff level of $\leq 70 \mathrm{ng} / \mathrm{ml}$ and PGI/PGII $\leq$ 3.0, the criterion recommended by Miki et al. [29], the sensitivity, specificity, and PPV were 68.4\% (95\% CI, $47.5 \%-89.3 \%), 85.6 \%$ (95\% CI, 84.9\%-86.2\%), and $0.7 \%$ (95\% CI, $0.34 \%-1.14 \%$ ), respectively. That is, the sensitivity was the same, the specificity was lower, and the PPV was slightly lower compared with the values for the direct X-ray examination. Moreover, there was a possibility that more gastric cancer cases, including cases that might not have been detected during the follow-up period or by X-ray, could have been diagnosed if this cutoff level had been used at the organization. Therefore, the PG test method alone, using the criteria of the organization, is not suitable for gastric cancer screening unless more valid criteria are used and/or the PG test is added to the X-ray method.

There are some limitations to our study. It is generally believed that intestinal-type early gastric cancer tends to progress slowly. As we did not carry out EGD for all participants who underwent gastric cancer screening, the possibility of false-negatives cannot be denied, even though follow up was done for 1 year. In addition, we could not cross-check gastric cancer cases diagnosed at institutes outside Osaka. Moreover, as the reported DCO rate of the Osaka Cancer Registry in 2000 was $24.3 \%$, this indicates that not all gastric cancer cases diagnosed prior to death were registered in the Osaka Cancer Registry.

Some researchers have pointed out the need to consider that although the PG method has an advantage in diagnosing intestinal-type gastric cancer derived from atrophic gastritis, the X-ray method has an advantage in diagnosing diffuse-type gastric cancer [30]. In fact, 
among the 19 gastric cancer cases detected in the present study, 8 cases were negative for the PG method, 2 were negative for the X-ray method, and 4 were negative for both. However, our data could not be used to indicate the appropriate combination of the two screening methods. To solve this problem, a prospective study, which can estimate the appropriate combination of the PG test method with a valid cutoff level and the X-ray method, setting the mortality rate as an end point, is needed in the future.

\section{Conclusion}

We conclude that the PG test method alone with a PGI cutoff level of $\leq 30 \mathrm{ng} / \mathrm{ml}$ and PGI/PGII $\leq 2.0$ is not an appropriate screening test for gastric cancer, as it showed a relatively lower level of sensitivity, a similar level of specificity, and a similar PPV compared with the values for the direct X-ray method. Considering the ease of PG measurement and the inefficiency of gastric radioscopy, it is desirable to study an effective combination of screening methods for gastric cancer, focusing on high risk populations.

Acknowledgments This work was supported in part by a Grant-in-Aid for Research from the Ministry of Health, Labour and Welfare, Japan (H16-025).

\section{References}

1. The Editorial Board of the Cancer Statistics in Japan. Cancer Statistics in Japan 2008. Tokyo: Foundation for Promotion of Cancer Research; 2008.

2. Oshima A, Hirata N, Ubukata T, Umeda K, Fujimoto I. Evaluation of a mass screening program for stomach cancer with a casecontrol study design. Int J Cancer 1986;38:829-33.

3. Fukao A, Tsubono Y, Tsuji I, Hisamichi S, Sugahara N, Takano A. The evaluation of screening for gastric cancer in Miyagi Prefecture, Japan: a population-based case-control study. Int J Cancer 1995;60:45-8.

4. Samloff IM, Varis K, Ihamaki T, Siurala M, Rotter JI. Relationships among serum pepsinogen I, serum pepsinogen II, and gastric mucosa histology. A study in relatives of patients with pernicious anemia. Gastroenterology 1982;83:204-9.

5. Miki K, Ichinose M, Shimizu A, Huang SC, Oka H, Furihata C, et al. Serum pepsinogens as a screening test of extensive chronic gastritis. Gastroenterol Jpn 1987;22:133-41.

6. Nomura AM, Stemmermann GN, Samloff IM. Serum pepsinogen I as a predictor of stomach cancer. Ann Intern Med 1980; 93:537-40.

7. Stemmermann GN, Samloff IM, Nomura AM, Heilbrun LK. Serum pepsinogens I and II and stomach cancer. Clin Chim Acta 1987;163:191-8.

8. Miki K, Ichinose M, Kawamura N, Matsushima M, Ahmad HB, Kimura M, et al. The significance of low serum pepsinogen levels to detect stomach cancer associated with extensive chronic gastritis in Japanese subjects. Jpn J Cancer Res 1989;80:111-4.
9. Miki K, Ichinose M, Ishikawa KB, Yahagi N, Matsushima M, Kakei N, et al. Clinical application of serum pepsinogen I and II levels for mass screening to detect gastric cancer. Jpn J Cancer Res 1993;84:1086-90.

10. Hamashima C, Shibuya D, Yamazaki H, Inoue K, Fukao A, Saito $\mathrm{H}$, et al. The Japanese guidelines for gastric cancer screening. Jpn J Clin Oncol 2008;38:259-67.

11. Miles A, Cockburn J, Smith RA, Wardle J. A perspective from countries using organized screening programs. Cancer 2004;101: 1201-13.

12. Statistics and Information Department, Ministry of Health, Labour, and Welfare. National reports on cancer screening programs 2004. Tokyo: Health and Welfare Statistics Association; 2006.

13. Murakami R, Tsukuma H, Ubukata T, Nakanishi K, Fujimoto I, Kawashima T, et al. Estimation of validity of mass screening program for gastric cancer in Osaka, Japan. Cancer 1990;65: 1255-60.

14. Sugawara N, Hirasawa Y, Morimoto T, Sibuki S, Kogane T, Sato $\mathrm{H}$, et al. An investigative report about health state of old age groups at first screening for gastric mass survey (in Japanese). J Gastroenterol Mass Surv 1992;95:184-6.

15. Fukao A, Hisamichi S, Takano A, Sugawara N. Accuracies of mass screening for gastric cancer-test sensitivity and program sensitivity (in Japanese). J Gastroenterol Mass Surv 1992;97: 59-63.

16. Ishida T, Suematsu T, Obayashi K, Takada Y, Kimura S, Suematsu C. Measurement of accuracy of stomach mass screening by population-based cancer registration (in Japanese). J Gastroenterol Mass Surv 1994;32:9-16.

17. Hattori M, Fujita M, Hosokawa O, Yamazaki S. A clinicopathological evaluation of false negative cases in gastric cancer mass survey (in Japanese). J Gastroenterol Mass Surv 1998; 36:468-75.

18. Abe S, Shibuya D, Noguchi T, Shimada T. An estimate of the false-negative rate of mass-screening for gastric carcinoma (in Japanese). J Gastroenterol Mass Surv 2000;38:475-82.

19. Sipponen P, Kekki M, Haapakoski J, Ihamaki T, Siurala M. Gastric cancer risk in chronic gastritis: statistical calculations of cross-sectional data. Int J Cancer 1985;35:173-7.

20. Correa P. Human gastric carcinogenesis: a multi-step and multifactorial process, First American Cancer Society Award Lecture on Cancer Epidemiology and Prevention. Cancer Res 1992;52: 6735-40.

21. Tatsuta M, Iishi H, Nakaizumi A, Okuda S, Taniguchi H, Hiyama $\mathrm{T}$, et al. Fundal atrophic gastritis as a risk factor for gastric cancer. Int J Cancer 1993;53:70-4.

22. Kimura K. Chronological transition of the fundic-pyloric border determined by stepwise biopsy of the lesser and greater curvatures of the stomach. Gastroenterology 1972;63:584-92.

23. Samloff IM. Cellular localization of group I pepsinogens in human gastric mucosa by immunofluorescence. Gastroenterology 1971; 61:185-8.

24. Samloff IM, Liebman WM. Cellular localization of the group II pepsinogens in human stomach and duodenum by immunofluorescence. Gastroenterology 1973;65:36-42.

25. Hattori Y, Tashiro H, Kawamoto T, Kodama Y. Sensitivity and specificity of mass screening for gastric cancer using the measurement of serum pepsinogens. Jpn J Cancer Res 1995;86:1210-5.

26. Kodoi A, Yoshihara M, Sumii K, Haruma K, Kajiyama G. Serum pepsinogen in screening for gastric cancer. J Gastroenterol 1995; 30:452-60.

27. Yoshihara M, Sumii K, Haruma K, Kiyohira K, Hattori N, Tanaka $\mathrm{S}$, et al. The usefulness of gastric mass screening using serum pepsinogen levels compared with photofluorography. Hiroshima J Med Sci 1997;46:81-6.

28. Kitahara F, Kobayashi K, Sato T, Kojima Y, Araki T, Fujino MA. Accuracy of screening for gastric cancer using serum pepsinogen concentrations. Gut 1999;44:693-7. 
29. Miki K, Morita M, Sasajima M, Hoshina R, Kanda E, Urita Y. Usefulness of gastric cancer screening using the serum pepsinogen test method. Am J Gastroenterol 2003;98: 735-9.
30. Ohata H, Oka M, Yanaoka K, Shimizu Y, Mukoubayashi C, Mugitani K, et al. Gastric cancer screening of a high-risk population in Japan using serum pepsinogen and barium digital radiography. Cancer Sci 2005;96:713-20. 